\title{
Effects of suprathermal electrons on the proton temperature anisotropy in space plasmas: Electromagnetic ion-cyclotron instability
}

\author{
S. M. Shaaban ${ }^{1,2} \bullet$ M. Lazar ${ }^{3,4} \cdot$ S. Poedts $^{1}$ • \\ A. Elhanbaly ${ }^{2}$
}

\begin{abstract}
In collision-poor plasmas from space, e.g., the solar wind and planetary magnetospheres, the kinetic anisotropy of the plasma particles is expected to be regulated by the kinetic instabilities. Driven by an excess of ion (proton) temperature perpendicular to the magnetic field $\left(T_{\perp}>T_{\|}\right)$, the electromagnetic ion-cyclotron (EMIC) instability is fast enough to constrain the proton anisotropy, but the observations do not conform to the instability thresholds predicted by the standard theory for bi-Maxwellian models of the plasma particles. This paper presents an extended investigation of the EMIC instability in the presence of suprathermal electrons which are ubiquitous in these environments. The analysis is based on the kinetic (Vlasov-Maxwell) theory assuming that both species, protons and electrons, may be anisotropic, and the EMIC unstable solutions are derived numerically providing an accurate description for conditions typically encountered in space plasmas. The effects of suprathermal populations are triggered by the electron anisotropy and the temperature contrast between electrons and protons. For certain conditions the anisotropy thresholds exceed the limits of the proton anisotropy measured in the solar wind considerably restraining the unstable regimes of the EMIC modes.
\end{abstract}

S. M. Shaaban

M. Lazar

S. Poedts

A. Elhanbaly

${ }^{1}$ Centre for Mathematical Plasma Astrophysics, Celestijnenlaan 200B, B-3001 Leuven, Belgium.

${ }^{2}$ Theoretical Physics Research Group, Physics Department, Faculty of Science, Mansoura University, 35516, Egypt.

${ }^{3}$ Royal Belgian Institute for Space Aeronomy 3-Avenue Circulaire, B-1180 Brussels, Belgium.

${ }^{4}$ School of Mathematics and Statistics, University of St Andrews, St Andrews, Fife, KY16 9SS, U.K.
Keywords plasmas - instabilities - solar wind

\section{INTRODUCTION}

Different physical mechanisms, which are at work in the solar wind and planetary magnetospheres, e.g., the solar wind expansion, compression of the magnetic field, may lead to large deviations from isotropy of the velocity distributions of plasma particles. Indeed, the in-situ measurements reveal the anisotropy of electrons and protons, which, for instance, exhibit different temperature components $T_{\perp} \neq T_{\|}$relative to the local magnetic field $\boldsymbol{B}_{\mathbf{0}}$ (Hellinger et al. 2006, Štverák et al. 12008). However, these deviations from isotropy are not large, and in the absence of collisions only the resulting instabilities may scatter particles back towards a quasi-equilibrium state and prevent the anisotropy to grow indefinitely (Gary and Lee 1994, Gary et al. 2001; Bale et al. 2009). Thus, in collisionless plasmas linear dispersion theory predicts that protons (ions) with an excess of perpendicular temperature $T_{p, \perp}>T_{p, \|}$ may drive the electromagnetic ion cyclotron (EMIC) instability (Gary 1993). According to Kennel and Petschek || (1966), this instability has a maximum growth rate at parallel propagation $\left(\boldsymbol{k} \times \boldsymbol{B}_{\mathbf{0}}=0\right)$, where the EMIC modes are left-hand ( $\mathrm{LH})$ circularly polarized.

In competition with mirror instability that develops for the same conditions, the EMIC modes grow faster (Gary et al. 1976), but the mirror thresholds shape better the limits of the proton anisotropy observed in the solar wind at $1 \mathrm{AU}$ (Hellinger et al. 2006). One possible explanation for this disagreement may reside in the fact that the EMIC fluctuations also dissipates faster due to resonant cyclotron interactions with protons (Bale et al. 2009). Otherwise, this disagreement may be a result of the limitations in the approach, which ignores either the interplay of protons with other 
species (e.g., alpha particles, electrons) or the presence of suprathermal (Kappa-like) populations. The presence of alpha particles changes the dispersive properties of the plasma and introduces the alpha cyclotron instability (Hellinger and Trávníček 2005). For certain conditions, e.g., protons and alpha particles with the same temperatures and temperature anisotropies, numerical simulations predict EMIC thresholds with a better alignment to the limits of proton anisotropy in the fast winds but not in the slow winds (Matteini et al. 2007). On the other hand, the instability conditions are found to be markedly influenced by the electrons with anisotropic temperatures, and their effect is enhanced by the electron-proton temperature ratio. However, the instability thresholds obtained in this case do not indicate better constrains for the proton anisotropy in the solar wind (Shaaban et al. 2015).

The electrons have an impact on proton instability by changing the wave phase velocity, which may increase the number of resonant protons and, implicitly, enhance the growth rate of the instability Kennel and Scarf 1968). Mutual effects of electrons and ions have already been studied for conditions favorable to the firehose instability Kennel and Scarf 1968. Michno et al. 2014) or the EMIC instability (Shaaban et al. 2015), but the influence of suprathermal populations was neglected in these cases. In the present paper we demonstrate the existence of new regimes of this instability triggered by the suprathermal electrons, which are ubiquitous in space plasmas. Enhanced by these suprathermal populations, the high energy tails of the electron velocity distribution functions (VDFs) measured in the solar wind (at various heliographic coordinates) are well described by the Kappa distribution functions (Vasyliunas 1968, Feldman et al. 1975 ; Maksimovic et al. 1997), which are nearly Maxwellian at low energies and decrease as power-laws at high energies. In the last decades, this realistic model has proved to be a veritable tool of modelling particle velocity distributions, replacing or complementing the standard Maxwellian distribution function when describing space pasma systems out of thermal equilibrium (Hellberg et al. 2005, 2009, Pierrard and Lazar 2010, Livadiotis and McComas 2013).

The EMIC instability conditions are therefore expected to be markedly altered by the deviations from thermodynamic equilibrium shown by the electron distributions in the solar wind, which may cumulate the effects of suprathermal populations and the anisotropic temperatures Maksimovic et al. 2005, Štverák et al. 2008). To include these effects in the analysis, we assume the electrons bi-Kappa distributed (Summers \& Thorne 1991), a model widely invoked to describe the kinetic instabilities in space plasmas (Mace et al. 2011 ; Kourakis et al. 2012; Lazar 2012, Henning and Mace |2014 Lazar et al. 2015). In Section 2 we introduce the distribution models for electrons and protons, and provide the dispersion relation for the EMIC modes. The unstable solutions as wave-number spectra of the growth rates and wave-frequencies are obtained numerically, enabling also to derive the anisotropy thresholds close to the marginal stability. In Section 3 we focus on the EMIC instability in the conditions relevant for the solar wind and planetary magnetospheres. The conclusions of the present study are presented in the last section.

\section{GOVERNING DISPERSION RELATION}

First, we introduce the models for the VDFs of the principal plasma components, the electrons (subscript $e$ ) and the protons (subscript $p$ ). In the unperturbed state the anisotropic protons are assumed bi-Maxwellian

$$
F_{p}\left(v_{\|}, v_{\perp}\right)=\frac{1}{\pi^{3 / 2} u_{p, \perp}^{2} u_{p, \|}} \exp \left(-\frac{v_{\|}^{2}}{u_{p, \|}^{2}}-\frac{v_{\perp}^{2}}{u_{p, \perp}^{2}}\right)
$$

where thermal velocities $u_{p, \|, \perp}$ are defined by the components of the anisotropic temperature

$$
\begin{aligned}
& T_{p, \|}^{M}=\frac{m}{k_{B}} \int d \mathbf{v} v_{\|}^{2} F_{p}\left(v_{\|}, v_{\perp}\right)=\frac{m u_{p, \|}^{2}}{2 k_{B}} \\
& T_{p, \perp}^{M}=\frac{m}{2 k_{B}} \int d \mathbf{v} v_{\perp}^{2} F_{p}\left(v_{\|}, v_{\perp}\right)=\frac{m u_{p, \perp}^{2}}{2 k_{B}} .
\end{aligned}
$$

Enhanced by the suprathermal populations, the anisotropic electron distributions are described by a biKappa VDF (Summers \& Thorne 1991)

$$
\begin{aligned}
F_{e}=\frac{1}{\pi^{3 / 2} \theta_{e, \perp}^{2} \theta_{e, \|}} & \frac{\Gamma\left(\kappa_{e}+1\right)}{\Gamma\left(\kappa_{e}-1 / 2\right)} \\
& \times\left[1+\frac{v_{\|}^{2}}{\kappa_{e} \theta_{e, \|}^{2}}+\frac{v_{\perp}^{2}}{\kappa_{e} \theta_{e, \perp}^{2}}\right]^{-\kappa_{e}-1},
\end{aligned}
$$

which is normalized to unity $\int d^{3} v F_{e}=1$, and is written in terms of thermal velocities $\theta_{e, \|, \perp}$ defined by the components of the effective temperature (for a powerindex $\kappa_{e}>3 / 2$ )

$T_{e, \|}^{K}=\frac{2 \kappa_{e}}{2 \kappa_{e}-3} \frac{m_{e}}{2 k_{B}} \theta_{e, \|}^{2}$

$T_{e, \perp}^{K}=\frac{2 \kappa_{e}}{2 \kappa_{e}-3} \frac{m_{e}}{2 k_{B}} \theta_{e, \perp}^{2}$ 
Without suprathermals, the electrons become biMaxwellian distributed $(\kappa \rightarrow \infty)$ and the components of their temperature reduce to

$\lim _{\kappa \rightarrow \infty} T_{e, \|, \perp}^{K}=\frac{m_{e}}{2 k_{B}} \theta_{e, \perp}^{2}=T_{e, \|, \perp}^{M}$.

In the presence of suprathermal populations quantified by a finite power-index $\kappa$, the electron temperature is enhanced (Lazar et al. 2015)

$T_{e, \|, \perp}^{K}=\frac{2 \kappa_{e}}{2 \kappa_{e}-3} T_{e, \|, \perp}^{M}>T_{e, \|, \perp}^{M}$,

and so is the electron plasma beta $\left(\beta_{e}=8 \pi n_{e} k_{B} T_{e} / B_{0}^{2}\right)$

$\beta_{e, \|, \perp}^{K}=\frac{2 \kappa_{e}}{2 \kappa_{e}-3} \beta_{e, \|, \perp}^{M}>\beta_{e, \|, \perp}^{M}$.

leading to the conclusion that dispersion properties and, implicitly, the instability conditions must also change. We have invetigated the effects of suprathermal populations on the plasma waves and instabilities using two alternative approaches, which assume the effective temperature to be either constant (Lazar et al. 2011, Mace et al. 2011; Lazar 2012), or increasing with the increase of suprathermal populations, i.e., with decreasing the power-index $\kappa$ (Luebner and Schupfer 2000 Lazar et al. 2015, 2016). Being more convenient computationally the approach with a $\kappa$-independent temperature has been widely invoked in similar theoretical predictions of plasma instabilities. However, Lazar et al. 2015) have recently shown and Lazar et al. (2016) have extended the comparative analysis to add supplementary arguments that a Kappa model with the effective temperature increasing with growing suprathermal population, i.e., with decreasing value of the power index $\kappa$, reproduces much better the Maxwellian core in the limit $\kappa \rightarrow \infty$, and thus enables a more realistic characterization of the suprathermal populations and their destabilizing effects.

For a collisionless and homogenous electron-proton plasma described by the distributions $(1)-(6)$, the dispersion relations for the electromagnetic modes propagating parallel to the stationary magnetic field read

$$
\begin{aligned}
& D^{ \pm}(k, \omega)=1-\frac{c^{2} k^{2}}{\omega^{2}}+\frac{\omega_{p, p}^{2}}{\omega^{2}}\left[\frac{\omega}{k u_{p, \|}} Z\left(\xi_{p}^{ \pm}\right)\right. \\
&\left.+\left(A_{p}-1\right)\left\{1+\xi_{p}^{ \pm} Z\left(\xi_{p}^{ \pm}\right)\right\}\right]+\frac{\omega_{p, e}^{2}}{\omega^{2}} \\
& \times\left[\frac{\omega}{k \theta_{\|, e}} Z_{\kappa}\left(g_{e}^{ \pm}\right)+\left(A_{e}-1\right)\right. \\
&\left.\times\left\{1+\frac{\omega \pm \Omega_{e}}{k \theta_{e, \|}} Z_{\kappa}\left(g_{e}^{ \pm}\right)\right\}\right]=0
\end{aligned}
$$

where $\omega$ is the wave-frequency, $k$ is the wave-number, $c$ is the speed of light in vacuum, $\Omega_{\alpha}=q_{\alpha} \mathbf{B}_{0} / m_{\alpha} c$ is the gyrofrequency (non-relativistic), $\omega_{p, \alpha}^{2}=4 \pi n_{\alpha} e^{2} / m_{\alpha}$ is the plasma frequency of different species $(\alpha=p, e)$, \pm denote, respectively, the circular right-handed $(\mathrm{RH})$ and left-handed ( $\mathrm{LH})$ polarization, and $A_{\alpha}=T_{\alpha, \perp} / T_{\alpha, \|}$ is the temperature anisotropy. In the direction parallel to the magnetic field, the EMIC modes decouples from the electrostatic oscillations, and their instability exhibits maximum growth rates (Kennel and Petschek (1966). The dispersion relation is obtained in terms of the plasma dispersion function (Fried and Conte 1961)

$$
Z\left(\xi_{p}^{ \pm}\right)=\frac{1}{\pi^{1 / 2}} \int_{-\infty}^{\infty} \frac{\exp \left(-x^{2}\right)}{x-\xi_{p}^{ \pm}} d t, \quad \Im\left(\xi_{p}^{ \pm}\right)>0,
$$

of argument

$\xi_{p}^{ \pm}=\frac{\omega \pm \Omega_{p}}{k u_{p, \|},}$

and the modified (Kappa) dispersion function (Lazar et al. 2008)

$$
\begin{aligned}
Z_{\kappa}\left(g_{e}^{ \pm}\right)= & \frac{1}{\pi^{1 / 2} \kappa_{e}^{1 / 2}} \frac{\Gamma\left(\kappa_{e}\right)}{\Gamma\left(\kappa_{e}-1 / 2\right)} \\
& \times \int_{-\infty}^{\infty} \frac{\left(1+x^{2} / \kappa_{e}\right)^{-\kappa_{e}}}{x-g_{e}^{ \pm}} d x, \quad \Im\left(g_{e}^{ \pm}\right)>0,
\end{aligned}
$$

of argument

$g_{e}^{ \pm}=\frac{\omega \pm \Omega_{e}}{k \theta_{\|, e}}$.

For the EMIC modes, which have LH polarization and $\omega<\Omega_{p}$, the dispersion relation from Eq. 10 can be rewritten with normalized quantities as follows

$$
\begin{aligned}
& \mu\left(A_{e}-1\right)+\mu \frac{A_{e} \tilde{\omega}+\mu\left(A_{e}-1\right)}{\tilde{k} \sqrt{\mu \Theta \beta_{p, \|}^{M}}} Z_{\kappa}\left(\frac{\tilde{\omega}+\mu}{\tilde{k} \sqrt{\mu \Theta \beta_{p, \|}^{M}}}\right) \\
& +A_{p}-1-\tilde{k}^{2}+\frac{A_{p}(\tilde{\omega}-1)+1}{\tilde{k} \sqrt{\beta_{p, \|}^{M}}} Z\left(\frac{\tilde{\omega}-1}{\tilde{k} \sqrt{\beta_{p, \|}^{M}}}\right)=0
\end{aligned}
$$

where $\tilde{\omega}=\omega / \Omega_{p}, \tilde{k}=k c / \omega_{p, p}, \mu=m_{p} / m_{e}$ is the proton/electron mass ratio, $\Theta=T_{e, \|}^{M} / T_{p, \|}^{M}$. is the electron/proton parallel temperature ratio in the Maxwellian limit for both species, and $\beta_{p, \|}^{M}=$ $8 \pi n_{e} k_{B} T_{p, \|}^{M} / B_{0}^{2}$ is the parallel proton beta parameter. 
(a) $\mathrm{A}_{\mathrm{p}}=2, \mathrm{~A}_{\mathrm{e}}=2, \beta_{\mathrm{p}, \|}^{\mathrm{M}}=1, \Theta=4$

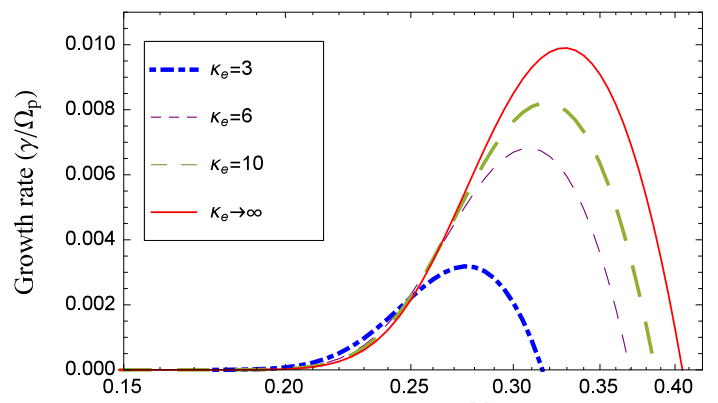

(b) $\mathrm{A}_{\mathrm{p}}=2, \mathrm{~A}_{\mathrm{e}}=1.5, \beta_{\mathrm{p}, \|}^{\mathrm{M}}=1, \Theta=4$

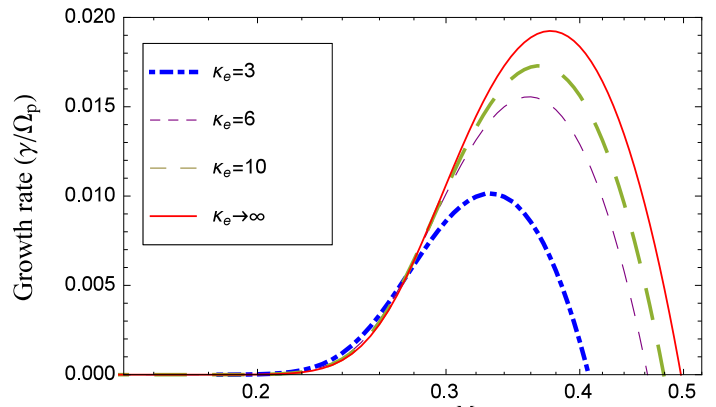

(c) $\mathrm{A}_{\mathrm{p}}=2, \mathrm{~A}_{\mathrm{e}}=1, \beta_{\mathrm{p}, \|}^{\mathrm{M}}=1, \Theta=4$

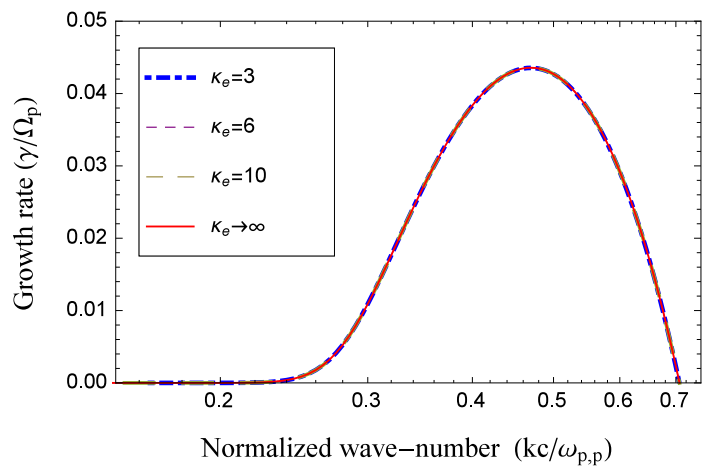

Fig. 1 Effects of the suprathermal electrons with $\kappa_{e}=3,6,10, \infty$ and $A_{e}=2$ (top), 1.5 (middle), 1 (bottom) on the growth rates of EMIC instability for $A_{p}=2$, $\beta_{p, \|}^{M}=1, \Theta=4$.

In the Maxwellian limit $\kappa \rightarrow \infty$ the dispersion relation (14) reduces exactly to Eq. (4) from Shaaban et al. (2015).

\section{RESULTS}

The exact solutions of the dispersion relation (14) are derived numerically, providing accurate description for the unstable EMIC modes. We investigate the effects of the suprathermal electrons on the instability for two distinct cases, complementary to each other, i.e., either when the electrons exhibit an excess of perpendicular temperature, i.e., $A_{e}>1$, or the opposite case, when the electrons are more energetic in direction parallel to the magnetic field, i.e., $A_{e}<1$. (a) $\mathrm{A}_{\mathrm{p}}=2, \mathrm{~A}_{\mathrm{e}}=2, \beta_{\mathrm{p}, \|}^{\mathrm{M}}=1, \Theta=4$

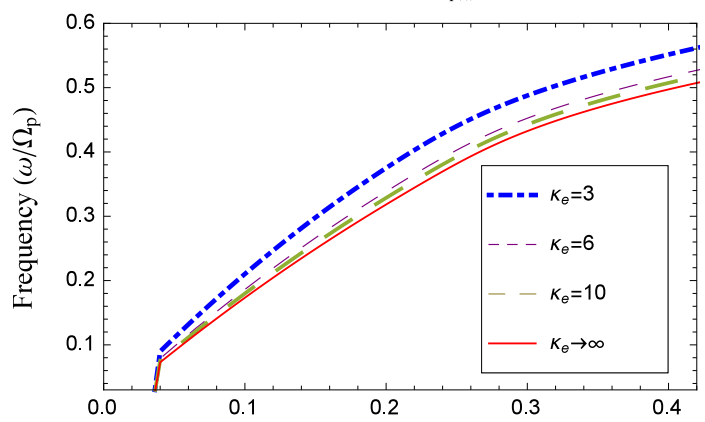

(b) $\mathrm{A}_{\mathrm{p}}=2, \mathrm{~A}_{\mathrm{e}}=1.5, \beta_{\mathrm{p}, \|}^{\mathrm{M}}=1, \Theta=4$

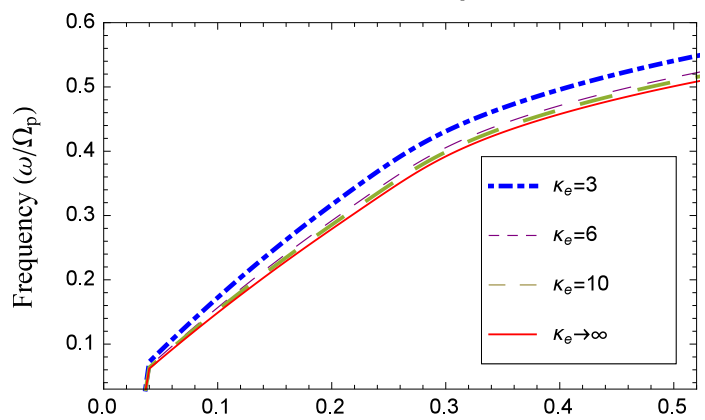

(c) $\mathrm{A}_{\mathrm{p}}=2, \mathrm{~A}_{\mathrm{e}}=1, \beta_{\mathrm{p}, \|}^{\mathrm{M}}=1, \Theta=4$

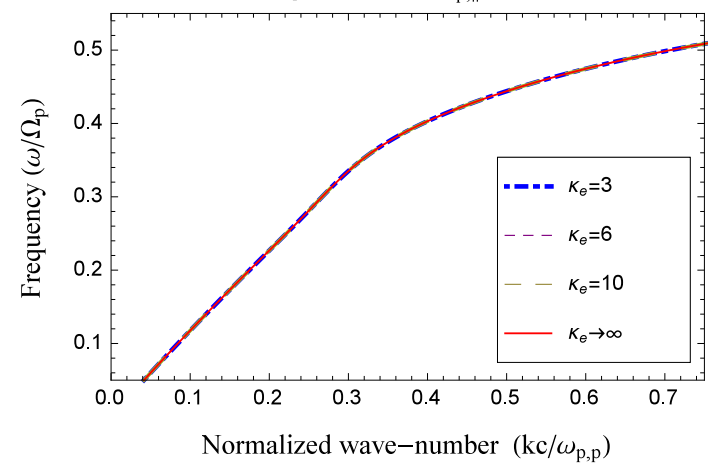

Fig. 2 Effects of the suprathermal electrons with $\kappa_{e}=3,6,10, \infty$ and $A_{e}=2$ (top), 1.5 (middle), 1 (bottom) on the wave-frequency of EMIC instability for $A_{p}=2$, $\beta_{p, \|}^{M}=1, \Theta=4$.

\subsection{Electrons with $A_{e}>1$}

We first examine the case when the electrons show an excess of transverse temperature, i.e., $T_{e, \perp}>T_{e, \|}$ (or $A_{e}>1$ ). In the absence of suprathermal populations, Shaaban et al. (2015) have found that the electrons with $A_{e}>1$ inhibit the EMIC instability and this effect is stimulated by the temperature ratio $\Theta$. Here we introduce a new parameter, namely, the power-index $\kappa_{e}$ that quantifies the presence and, implicitly, the effects of the suprathermal electrons. Figs. 1 and 2 display the wave-number dispersion for the growth rate, and the wave-frequency, respectively, for the same set of parameters $A_{p}=2, \beta_{p, \|}^{M}=1$, and $\Theta=4$, but different kappa indices $\kappa_{e}=3,6,10, \infty$ and different electron 
(a) $\mathrm{A}_{\mathrm{p}}=2, \mathrm{~A}_{\mathrm{e}}=1.5, \beta_{\mathrm{p} \|}^{\mathrm{M}}=1, \Theta=4$

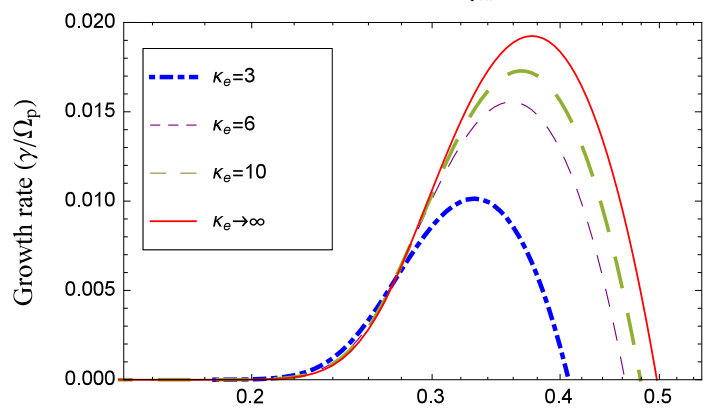

(b) $\mathrm{A}_{\mathrm{p}}=2, \mathrm{~A}_{\mathrm{e}}=1.5, \beta_{\mathrm{p}, \|}^{\mathrm{M}}=1, \Theta=2$

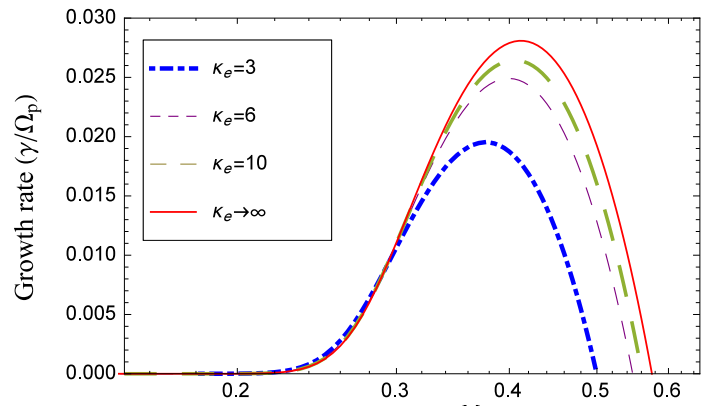

(c) $\mathrm{A}_{\mathrm{p}}=2, \mathrm{~A}_{\mathrm{e}}=1.5, \beta_{\mathrm{p}, \|}^{\mathrm{M}}=1, \Theta=1$

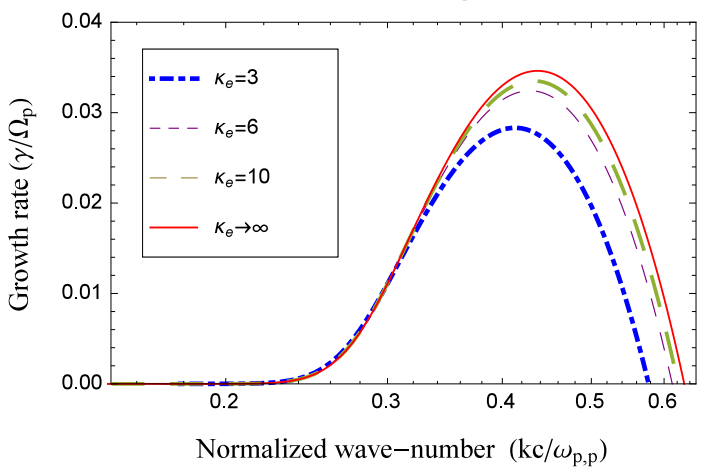

Fig. 3 Effects of the suprathermal electrons with $\kappa_{e}=3,6,10, \infty$ and the temperature ratio $\Theta=4$ (top), 2 (middle), 1 (bottom) on the growth rates of EMIC instability for $A_{p}=2, A_{e}=1.5, \beta_{p, \|}^{M}=1$.

anisotropies $A_{e}=1,1.5,2$. In Figs. 3 and 4 we keep constant the electron anisotropy $A_{e}=1.5$ but vary the electron/proton temperature ratio $\Theta=1,2,4$. All these values are chosen according to the observations in the solar wind (Štverák et al. 2008, Newbury et al. 1998).

The inhibiting effect of the electron anisotropy on the EMIC instability is reconfirmed by the unstable solutions displayed in Fig. 1. In addition, the same figure shows that this effect may be significantly enhanced by the suprathermal electrons, namely, the growth-rate peaks markedly decrease with decreasing the powerindex $\kappa_{e}$. For isotropic electrons, i.e., panels (c) in both Figs. 1 and 2, the suprathermal populations do not have any influence on the EMIC instability. On the other hand, from a comparison with Fig. 3 we can (a) $\mathrm{A}_{\mathrm{p}}=2, \mathrm{~A}_{\mathrm{e}}=1.5, \beta_{\mathrm{p}, \|}^{\mathrm{M}}=1, \Theta=4$

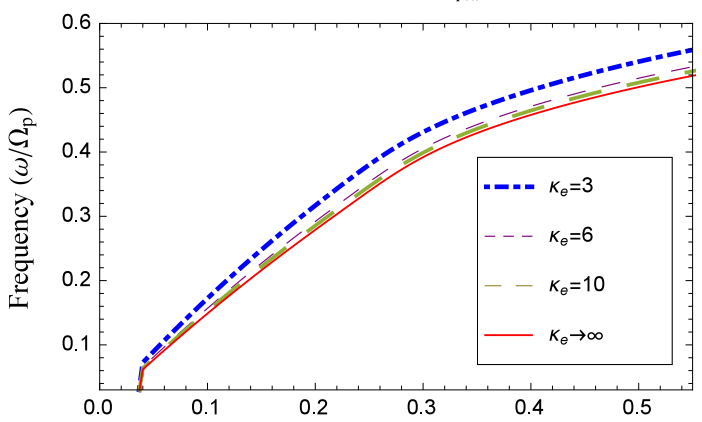

(b) $\mathrm{A}_{\mathrm{p}}=2, \mathrm{~A}_{\mathrm{e}}=1.5, \beta_{\mathrm{p}, \|}^{\mathrm{M}}=1, \Theta=2$

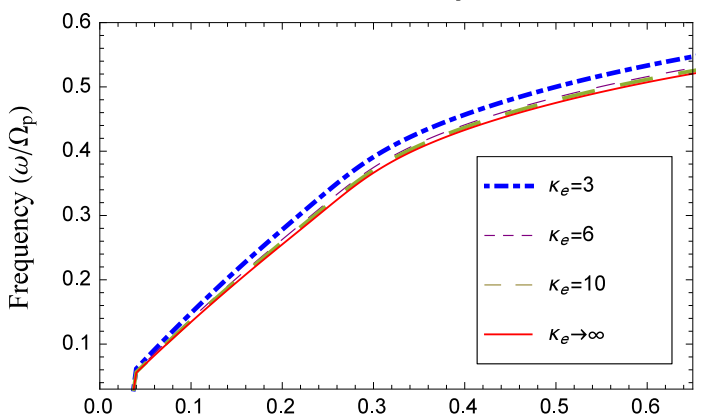

(c) $\mathrm{A}_{\mathrm{p}}=2, \mathrm{~A}_{\mathrm{e}}=1.5, \beta_{\mathrm{p}, \|}^{\mathrm{M}}=1, \Theta=1$

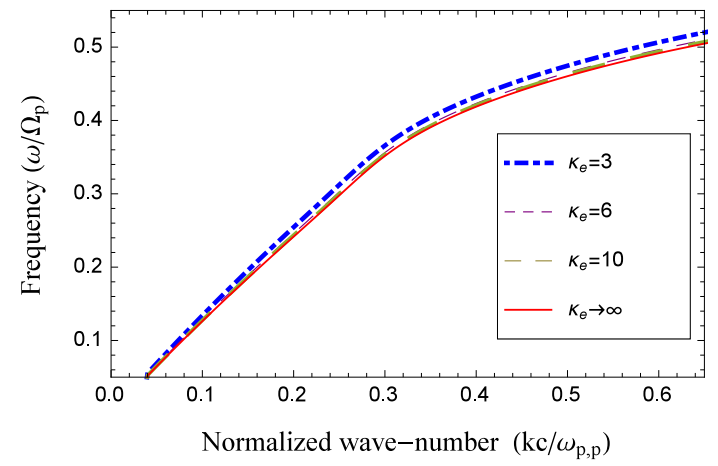

Fig. 4 Effects of the suprathermal electrons with $\kappa_{e}=3,6,10, \infty$ and the temperature ratio $\Theta=4$ (top), 2 (middle), 1 (bottom) on the wave-frequency of EMIC instability for $A_{p}=2, A_{e}=1.5, \beta_{p, \|}^{M}=1$.

observe that the EMIC instability is inhibited by increasing the temperature contrast $\Theta$ between electrons and protons, and again, this effect is stimulated by the suprathermal electrons (i.e., decreasing the power-index $\left.\kappa_{e}\right)$. The wave-frequency in Figs. 2 and 4 show an opposite tendency, with values being slightly diminished by these effects.

For the sake of comparison, we have also studied the influence of the suprathermal electrons on the EMIC instability in the alternative approach which assumes the temperature independent of kappa index. Fig. 5 displays unstable solutions representative for this approach, and these solutions show a negligible influence of the suprathermal electrons. We have found the same 
(a) $\mathrm{A}_{\mathrm{p}}=2, \mathrm{~A}_{\mathrm{e}}=1.5, \beta_{\mathrm{p}, \|}^{\mathrm{M}}=1, \Theta=4$

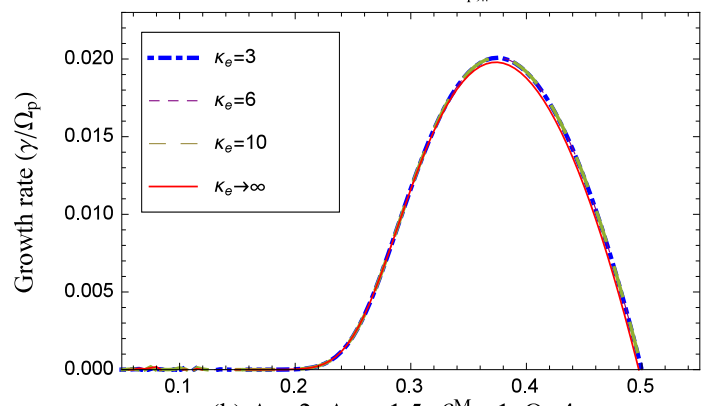

(b) $\mathrm{A}_{\mathrm{p}}=2, \mathrm{~A}_{\mathrm{e}}=1.5, \beta_{\mathrm{p}, \|}^{\mathrm{M}}=1, \Theta=4$

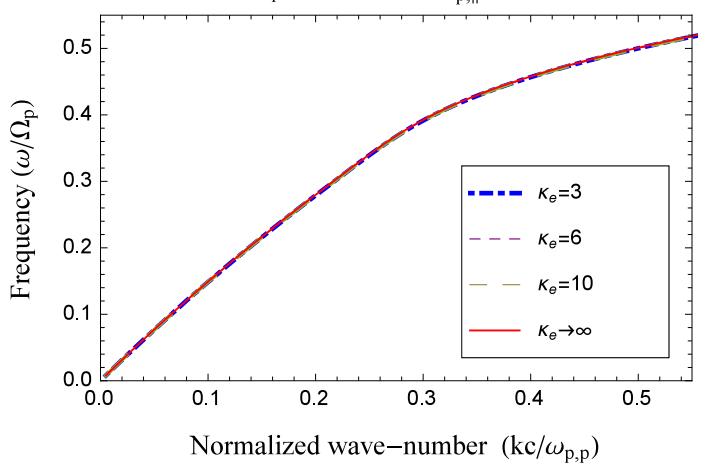

Fig. 5 Effects of the suprathermal electrons with $\kappa_{e}=3,6,10, \infty$ on the growth rates (a) and wave-frequency (b) of EMIC instability for $A_{p}=2, A_{e}=1.5, \beta_{p, \|}^{M}=1$, $\Theta=4$.

insignificant influence on the instability thresholds, and so, there is no need to plot them here.

Finding a systematic inhibition of the EMIC instability under the effect of suprathermal electrons, determined us to re-evaluate the anisotropy thresholds of this instability in the new conditions, and compare them with the limits of the proton anisotropy observed in the solar wind. The anisotropy thresholds for the EMIC instability are provided by the dispersion relation for low levels of maximum growth-rates $\gamma_{\mathrm{m}} / \Omega_{p}=10^{-2}, 10^{-3}$, approaching marginal condition of stability $\left(\gamma_{\mathrm{m}}=0\right)$. In Figs. 6 and 7 the anisotropy thresholds are calculated for an extended range of the plasma beta parameter $0.01<\beta_{p, \|}^{M}<100$, which includes conditions typical for the solar wind and terrestrial magnetosphere. In Fig. 6 we keep constant the temperature ratio $\Theta=4$, but vary the electron anisotropy $A_{e}=2,1.5,1$ and the power-index $\kappa_{e}=2,6, \infty$. Contours of the maximum growth rates $\gamma_{\mathrm{m}} / \Omega_{p}=10^{-3}$ are fitted to an inverse correlation law between the temperature anisotropy, $A_{p}$, and the parallel plasma beta $\beta_{p, \|}^{M}$ (Gary and Lee 1994)

$$
A_{p}=1+\frac{a}{\beta_{p, \|}^{M^{b}}}
$$

Table 1 Fitting parameters for the proton anisotropy in Eq. (15)

\begin{tabular}{cccc}
\hline \hline & \multicolumn{3}{c}{$\gamma_{\mathrm{m}} / \Omega=10^{-3}$} \\
$\kappa_{e}$ & $A_{e}$ & $a$ & $b$ \\
\hline 2 & 2 & 1.4043 & 0.1390 \\
& 1.5 & 1.0852 & 0.1952 \\
& 1 & 0.4504 & 0.4020 \\
6 & 2 & 0.7624 & 0.2785 \\
& 1.5 & 0.6295 & 0.3235 \\
& 1 & 0.4504 & 0.4020 \\
$\infty$ & 2 & 0.7028 & 0.2975 \\
& 1.5 & 0.5920 & 0.3382 \\
& 1 & 0.4504 & 0.4020 \\
\hline
\end{tabular}

Table 2 Fitting parameters for the proton anisotropy in Eq. (15)

\begin{tabular}{cccc}
\hline \hline & \multicolumn{3}{c}{$\gamma_{\mathrm{m}} / \Omega=10^{-2}$} \\
$\Theta$ & $\kappa_{e}$ & $a$ & $b$ \\
\hline 4 & 2 & 1.7233 & 0.1742 \\
& 6 & 1.0752 & 0.2840 \\
& $\infty$ & 0.9903 & 0.3038 \\
2 & 2 & 1.2980 & 0.2404 \\
& 6 & 0.8873 & 0.3304 \\
& $\infty$ & 0.8352 & 0.3448 \\
1 & 2 & 1.01818 & 0.2966 \\
& 6 & 0.7784 & 0.3612 \\
& $\infty$ & 0.7464 & 0.3711 \\
\hline
\end{tabular}

where the fitting parameters $a$ and $b$ are tabulated in Table 1. The anisotropy thresholds plotted in Fig. 6 are increased by the electron anisotropy and this difference becomes more pronounced for higher values of the plasma beta parameter. These effects are found to be markedly enhanced by the suprathermal populations (i.e., decreasing the power-index $\kappa_{e}$ ) confirming the influence shown already in Figs. 1 and 3 on the instability growth-rates.

In Fig. 7 we keep constant the electron anisotropy $A_{e}=2$ and study the effect of the power-index $\kappa_{e}=$ $2,6, \infty$ and the temperature ratio $\Theta=4,2,1$ on the anisotropy thresholds derived for $\gamma_{\mathrm{m}} / \Omega_{p}=10^{-2}$. Fitting parameters $a$ and $b$ are tabulated in Table 2. The effect of suprathermal electrons on the EMIC thresholds increases with the temperature ratio $\Theta$, and this effect is still apparent for isothermal species with $\Theta=1$.

In both cases from Figs. 6 and 7 the anisotropy thresholds are compared with the limits of the proton anisotropy from the 10-year measurements at $1 \mathrm{AU}$ in the solar wind (Bale et al. 2009: Shaaban et al. 2015). We use the observational data from WIND/SWE and MFI instruments for the proton velocity distribution 

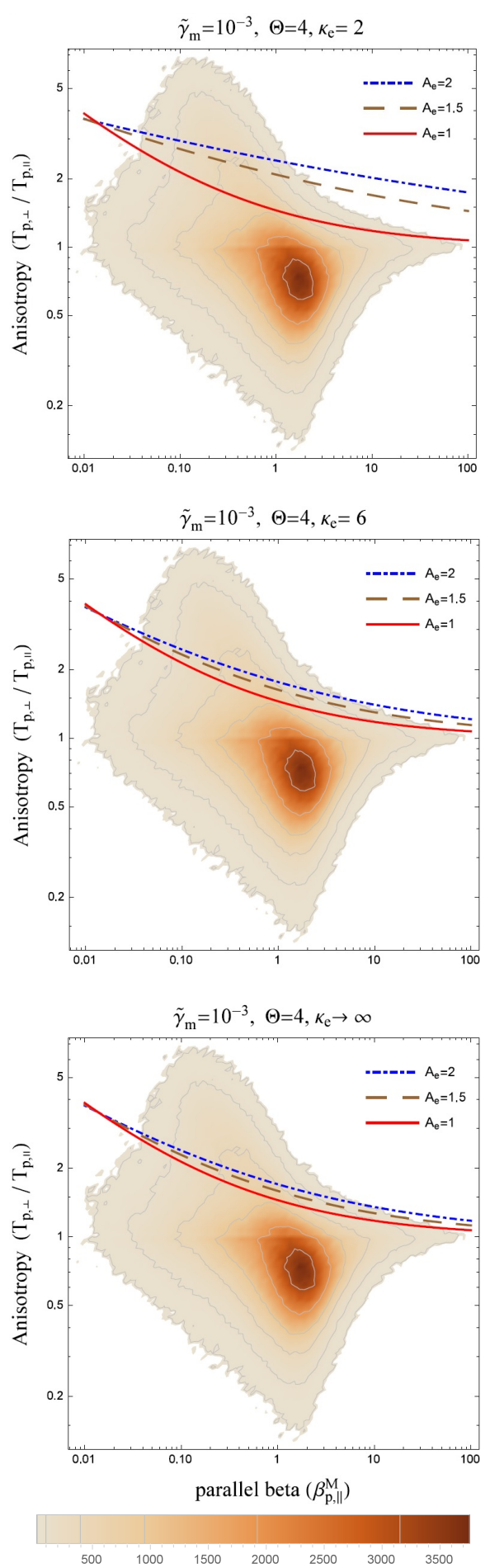

Fig. 6 Effects of the suprathermal electrons with $A_{e}=2,1.5,1$ and $\kappa_{e}=2$ (top), 6 (middle), $\infty$ (bottom) on the threshold conditions of EMIC instability with the maximum growth rate $\gamma_{m} / \Omega_{p}=10^{-3}$ for $\Theta=4$.
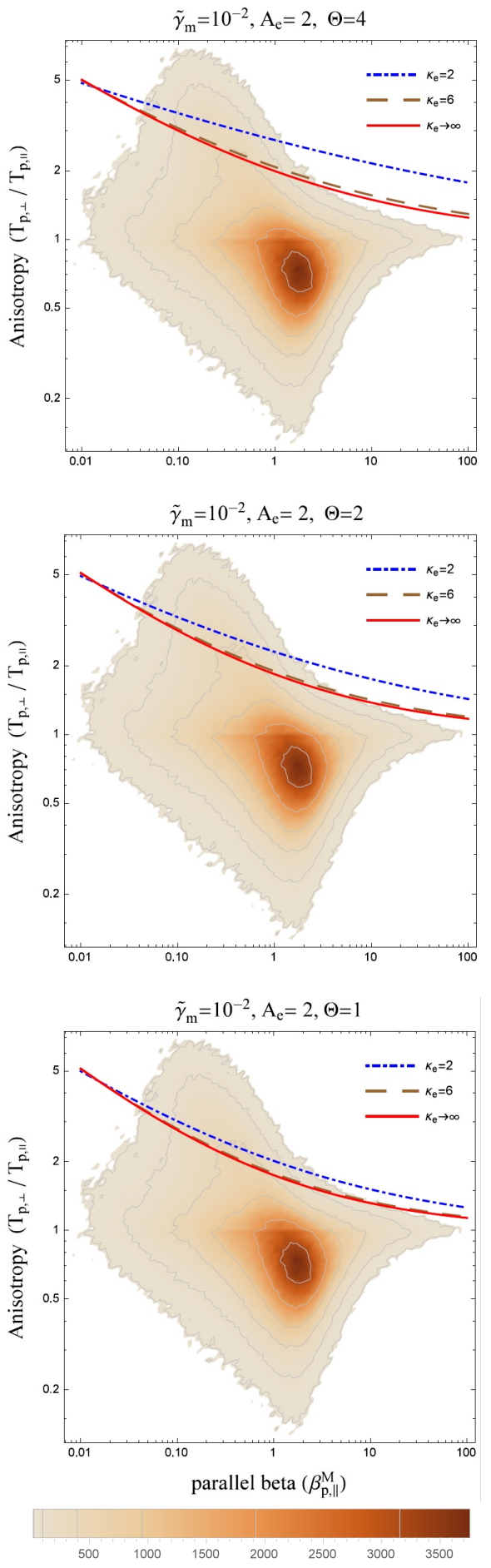

Fig. 7 Effects of the suprathermal electrons with $\kappa_{e}=2,6, \infty$ and the electron/proton temperature ratio $\Theta=4$ (top), 2 (middle), 1 (bottom) on the threshold conditions of EMIC instability with the maximum growth rate $\gamma_{m} / \Omega_{p}=10^{-2}$ for $A_{e}=2$. 
(a) $\mathrm{A}_{\mathrm{p}}=2, \mathrm{~A}_{\mathrm{e}}=0.55, \beta_{\mathrm{p}, \|}^{\mathrm{M}}=1, \Theta=4$

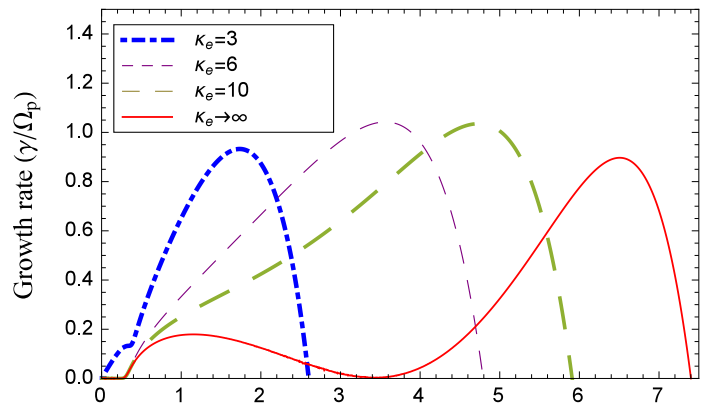

(b) $\mathrm{A}_{\mathrm{p}}=2, \mathrm{~A}_{\mathrm{e}}=0.6, \beta_{\mathrm{p}, \|}^{\mathrm{M}}=1, \Theta=4$

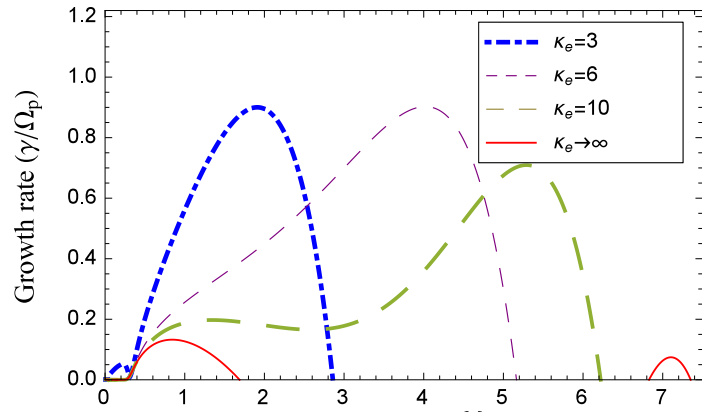

(c) $\mathrm{A}_{\mathrm{p}}=2, \mathrm{~A}_{\mathrm{e}}=0.65, \beta_{\mathrm{p}, \|}^{\mathrm{M}}=1, \Theta=4$

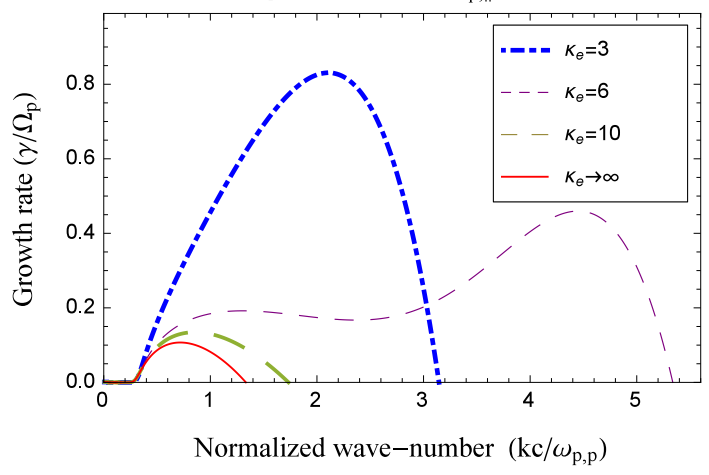

Fig. 8 Effects of the suprathermal electrons with $\kappa_{e}=3,6,10, \infty$ and $A_{e}=0.55$ (top), 0.6 (middle), 0.65 (bottom) on the growth rates of EMIC instability for $A_{p}=2$, $\beta_{p, \|}^{M}=1, \Theta=4$.

and magnetic field, respectively (Lepping et al. 1995 Ogilvie et al. 1995). The solar wind proton anisotropy is displayed with a color logarithmic scale representing the number of events (only for bins with more than 20 events). According to our discussion in the introduction, the EMIC instability is expected to constrain the proton anisotropy in the solar wind, with instability thresholds aligned to (Gary et al. 2001) or exceeding (Isenberg et al. 2013) the limits of the proton anisotropy observed in the solar wind. In these new regimes investigated here, the electrons with anisotropic temperature and an important suprathermal component may have an important effect on the EMIC instability, inhibiting the growth-rates and increasing the thresholds, especially when the instabil- (a) $\mathrm{A}_{\mathrm{p}}=2, \mathrm{~A}_{\mathrm{e}}=0.55, \beta_{\mathrm{p}, \|}^{\mathrm{M}}=1, \Theta=4$

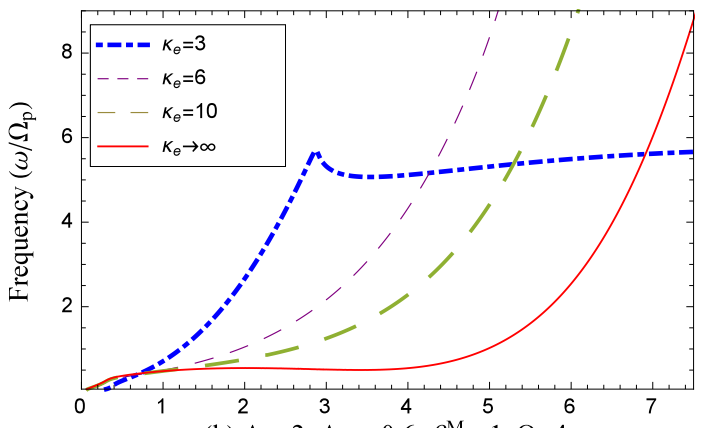

(b) $\mathrm{A}_{\mathrm{p}}=2, \mathrm{~A}_{\mathrm{e}}=0.6, \beta_{\mathrm{p}, \|}^{\mathrm{M}}=1, \Theta=4$

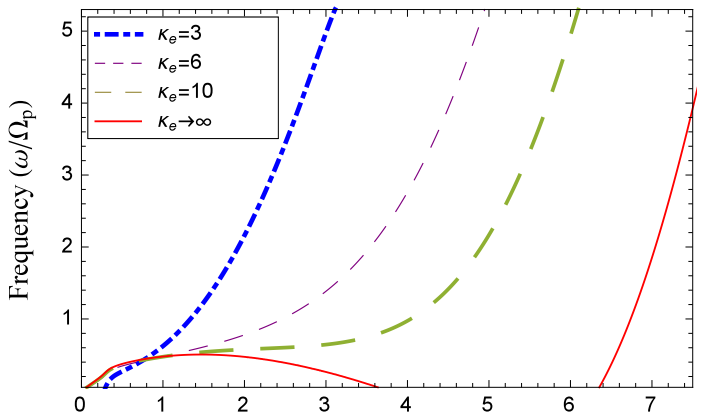

(c) $\mathrm{A}_{\mathrm{p}}=2, \mathrm{~A}_{\mathrm{e}}=0.65, \beta_{\mathrm{p}, \|}^{\mathrm{M}}=1, \Theta=4$

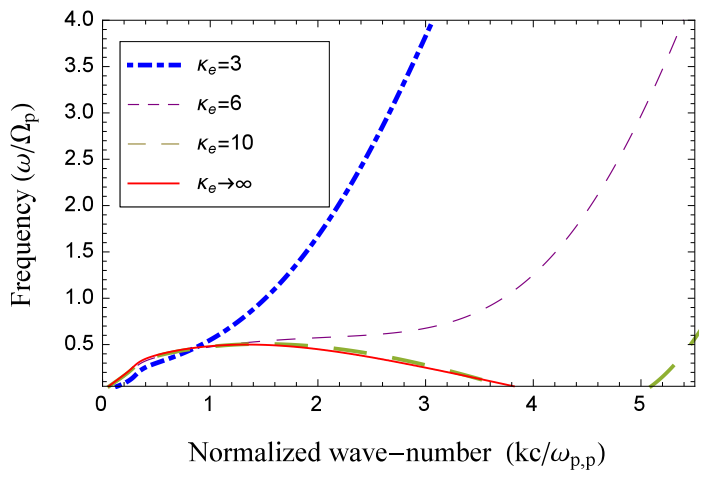

Fig. 9 Effects of the suprathermal electrons with $\kappa_{e}=3,6,10, \infty$ and $A_{e}=0.55$ (top), 0.6 (middle), 0.65 (bottom) on the wave-frequency of EMIC instability for $A_{p}=2$, $\beta_{p, \|}^{M}=1, \Theta=4$.

ity is triggered by highly hot and anisotropic protons. However, the new EMIC thresholds obtained here do not show a good fit to the observations, but may rather exceed the limits of the proton anisotropy for large enough values of the plasma beta $\beta_{p, \|}^{M}$.

\subsection{Electrons with $A_{e}<1$}

In the second part of this section we describe the influence of suprathermal electrons on the EMIC instability in the opposite case, namely, when $T_{e, \|}>T_{e, \perp}$. Figs. 8 and 9 display the wave-number dispersion of the growth rate and the wave-frequency, respectively, for the same set of parameters $A_{p}=2, \beta_{p, \|}^{M}=1$, and $\Theta=4$, but different kappa indices $\kappa_{e}=3,6,10, \infty$ and electron 
(a) $\mathrm{A}_{\mathrm{p}}=2, \mathrm{~A}_{\mathrm{e}}=0.75, \beta_{\mathrm{p}, \|}^{\mathrm{M}}=2, \Theta=4$

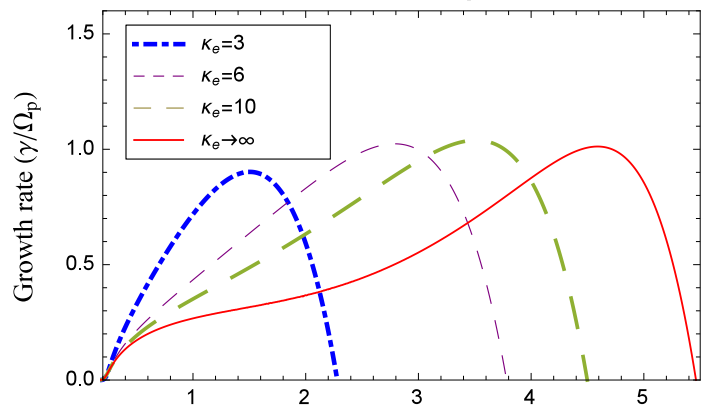

(b) $\mathrm{A}_{\mathrm{p}}=2, \mathrm{~A}_{\mathrm{e}}=0.75, \beta_{\mathrm{p}, \|}^{\mathrm{M}}=2, \Theta=3$

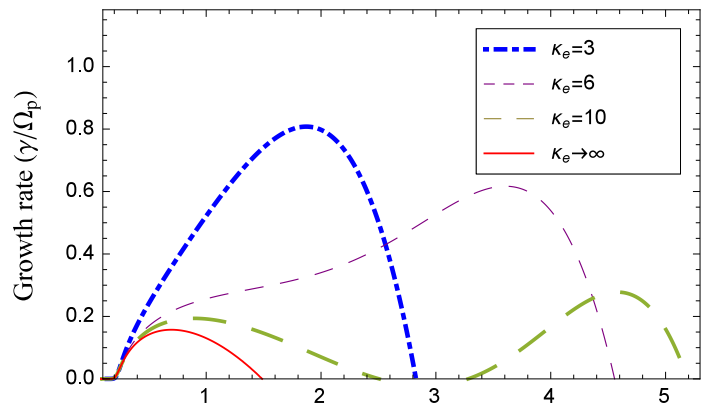

(c) $\mathrm{A}_{\mathrm{p}}=2, \mathrm{~A}_{\mathrm{e}}=0.75, \beta_{\mathrm{p}, \|}^{\mathrm{M}}=2, \Theta=1$

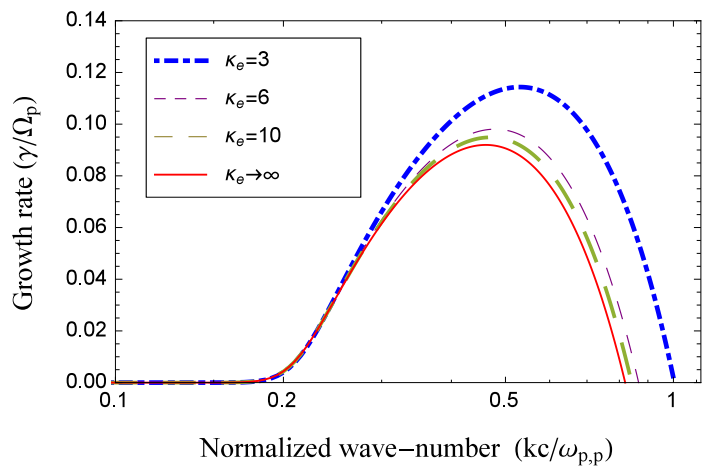

Fig. 10 Effects of the suprathermal electrons with $\kappa_{e}=3,6,10, \infty$ and the temperature ratio $\Theta=4$ (top), 3 (middle), 1 (bottom) on the growth rates of EMIC instability for $A_{p}=2, A_{e}=0.75, \beta_{p, \|}^{M}=2$.

anisotropies $A_{e}=0.55,0.6,0.65$. In Figs. 10 and 11 we keep constant the electron anisotropy $A_{e}=0.75$ but vary the electron/proton temperature ratio $\Theta=1,3,4$.

In Figs. 8 and 10 the growth rates of the unstable solutions may display two distinct peaks, one at low wave-numbers corresponding to the EMIC mode, and a second peak arising at higher wave-numbers due to the electron firehose (EFH) instability. Both these two modes have the same LH circular polarization, which is also confirmed by the same positive sign of their wavefrequencies in Figs. 9 and 11. The existence and the dominance of the second peak depend on both the electron anisotropy $A_{e}$ and the power index $\kappa_{e}$. In the presence of suprathermal electrons the EFH becomes dominant with a peak much higher than the EMIC, (a) $\mathrm{A}_{\mathrm{p}}=2, \mathrm{~A}_{\mathrm{e}}=0.75, \beta_{\mathrm{p}, \|}^{\mathrm{M}}=2, \Theta=4$

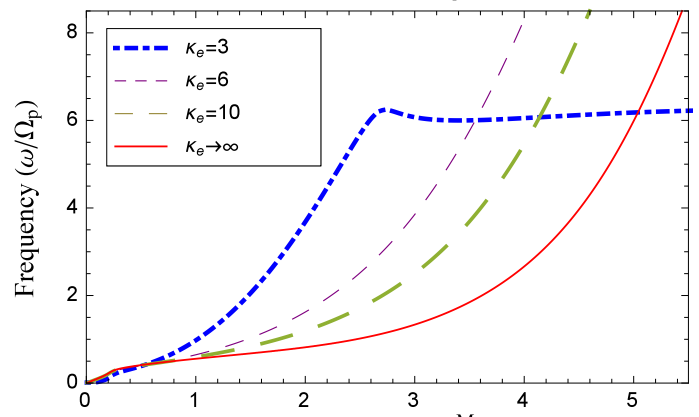

(b) $\mathrm{A}_{\mathrm{p}}=2, \mathrm{~A}_{\mathrm{e}}=0.75, \beta_{\mathrm{p}, \|}^{\mathrm{M}}=2, \Theta=3$

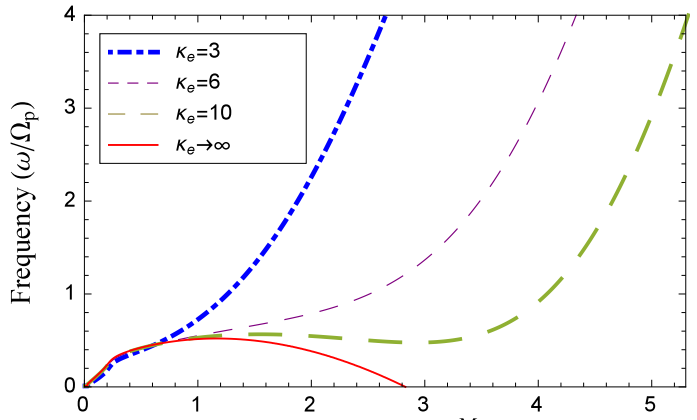

(c) $\mathrm{A}_{\mathrm{p}}=2, \mathrm{~A}_{\mathrm{e}}=0.75, \beta_{\mathrm{p}, \|}^{\mathrm{M}}=2, \Theta=1$

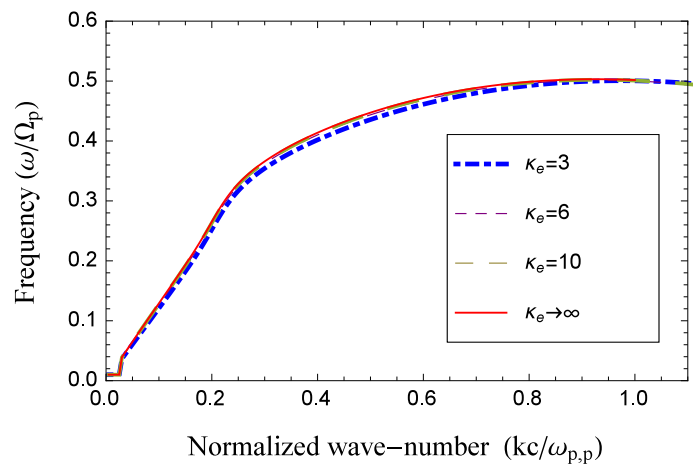

Fig. 11 Effects of the suprathermal electrons with $\kappa_{e}=3,6,10, \infty$ and the temperature ratio $\Theta=4$ (top), 3 (middle), 1 (bottom) on the wave-frequency of EMIC instability for $A_{p}=2, A_{e}=0.75, \beta_{p, \|}^{M}=2$.

even for small small deviations of the electrons from isotropy. For instance, for the lowest values of the power-index $\kappa_{e}=3,6$ in panels (a) and (b), the EMIC peak can only hardly be distinguished. The unstable solutions obtained in the absence of suprathermal electrons $\left(\kappa_{e} \rightarrow \infty\right)$ are plotted with solid lines, and their dependence on the electron anisotropy and the electron/proton temperature ratio is similar to that described by Shaaban et al. (2015).

Another factor that stimulates the EFH instability is the electron/proton temperature ratio $\Theta$ and for higher values $\Theta=3,4$ the EMIC peak is completely hidden by the EFH peak, see in Fig. 10, panels (a) and (b). However, the effect of the suprathermal electrons on the EMIC instability (first peak) appears more clear in 
(a) $\mathrm{A}_{\mathrm{p}}=2, \mathrm{~A}_{\mathrm{e}}=0.55, \beta_{\mathrm{p}, \|}^{\mathrm{M}}=1, \Theta=4$

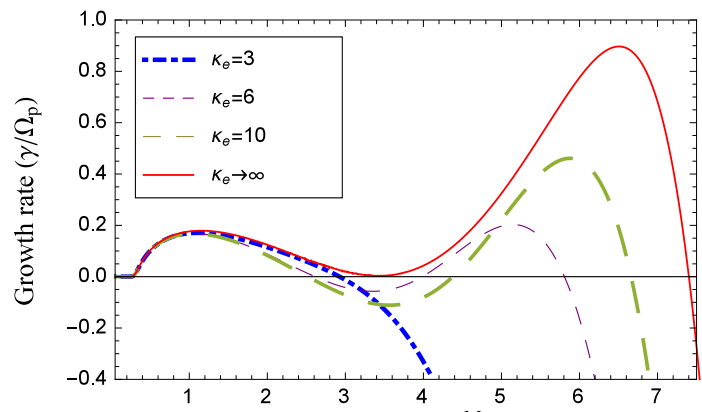

(b) $\mathrm{A}_{\mathrm{p}}=2, \mathrm{~A}_{\mathrm{e}}=0.6, \beta_{\mathrm{p}, \|}^{\mathrm{M}}=1, \Theta=4$

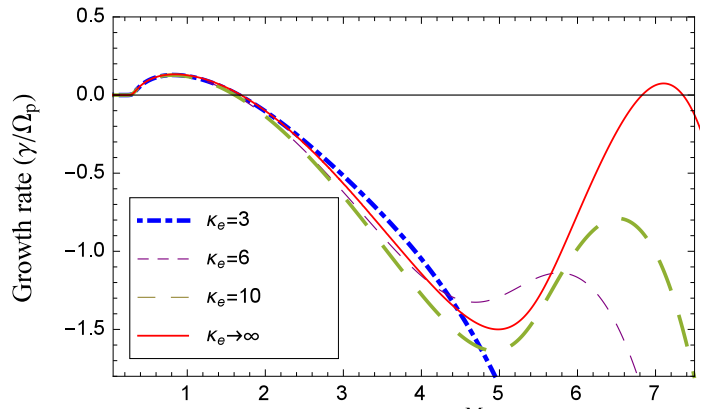

(c) $\mathrm{A}_{\mathrm{p}}=2, \mathrm{~A}_{\mathrm{e}}=0.65, \beta_{\mathrm{p}, \|}^{\mathrm{M}}=1, \Theta=4$

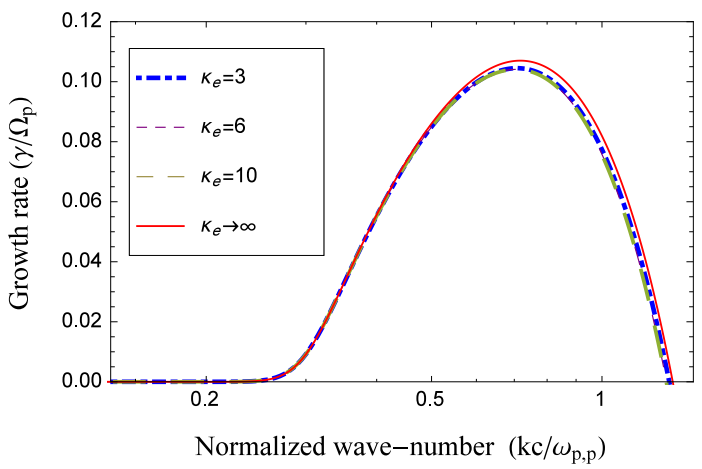

Fig. 12 Effects of the suprathermal electrons with $\kappa_{e}=3,6,10, \infty$ and $A_{e}=0.55$ (top), 0.6 (middle), 0.65 (bottom) on the growth rates of EMIC instability for $A_{p}=2$, $\beta_{p, \|}^{M}=1, \Theta=4$.

panel (c), when the temperature contrast is minimized $(\Theta=1)$ and the deviation from isotropy is sufficiently small $\left(A_{e}=0.75\right)$. A detailed analysis of the EMIC thresholds by comparison to the observations in the solar wind is difficult to perform in this case, especially when the EMIC peak cannot be distinguished from the EFH peak (see also the explanations in Shaaban et al. [(2015)). When the EMIC peak is distinguishable it is found to be always stimulated by the suprathermal electrons with $A_{e}<1$, giving us possibility to conclude that the EMIC thresholds are lowered and more deviated from the anisotropy limits of solar wind protons.

Corresponding to these two instabilities, the wavefrequency displayed in Figs. 9 and 11 shows similar important variations function of the electron anisotropy, (a) $\mathrm{A}_{\mathrm{p}}=2, \mathrm{~A}_{\mathrm{e}}=0.55, \beta_{\mathrm{p}, \|}^{\mathrm{M}}=1, \Theta=4$

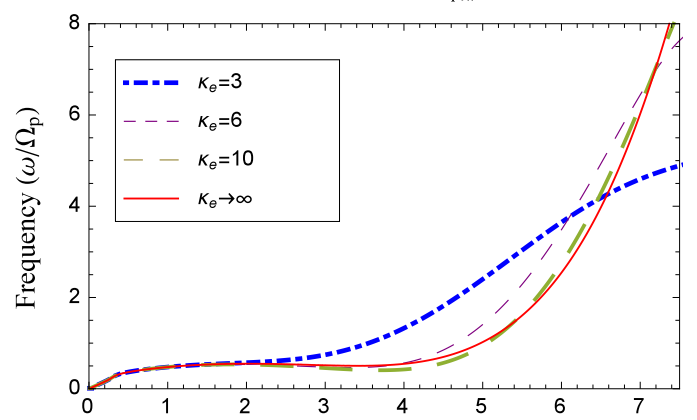

(b) $\mathrm{A}_{\mathrm{p}}=2, \mathrm{~A}_{\mathrm{e}}=0.6, \beta_{\mathrm{p}, \|}^{\mathrm{M}}=1, \Theta=4$

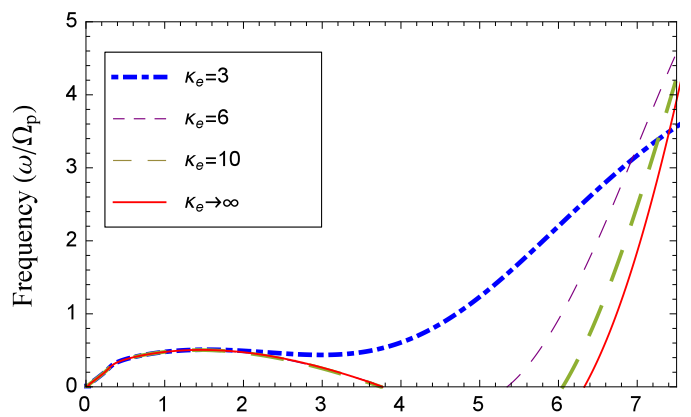

(c) $\mathrm{A}_{\mathrm{p}}=2, \mathrm{~A}_{\mathrm{e}}=0.65, \beta_{\mathrm{p}, \|}^{\mathrm{M}}=1, \Theta=4$

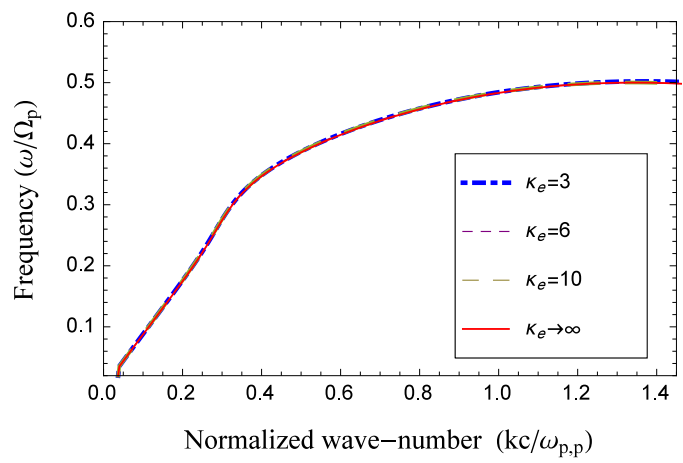

Fig. 13 Effects of the suprathermal electrons with $\kappa_{e}=3,6,10, \infty$ and $A_{e}=0.55$ (top), 0.6 (middle), 0.65 (bottom) on the wave-frequency of EMIC instability for $A_{p}=2$, $\beta_{p, \|}^{M}=1, \Theta=4$.

the power index $\kappa_{e}$ and the temperature contrast between electrons and protons. For isothermal components, i.e., when $\Theta=1$, these variations are negligibly small. These new results complement those obtained by Lazar et al. (2011) and Michno et al. (2014) in studies of the firehose instability cumulatively driven by the anisotropic protons and electrons, when both species manifest an excess of parallel temperature $\left(T_{\|}>T_{\perp}\right)$. In that case the unstable solutions show growth rates with two distinct peaks corresponding to the proton firehose and electron firehose instabilities, and the wave-frequency changes its sign according to the opposite polarizations of these two modes.

We have studied the same plasma conditions invoked in Figs. 8 and 9 in the alternative approach 
which assumes the effective temperature of the electrons independent of kappa index. For the unstable solutions derived in this case, a number of representative cases are displayed in Figs. 12 and 13 , showing the growth-rate and wave-frequency, respectively. In Figs. 12 we find the EFH peaks (at larger wave numbers) significantly inhibited by the suprathermal electrons, while the EMIC peaks (at lower wave-numbers) are not affected. Moreover, with decreasing the electron anisotropy the second peak is completely suppressed, and only the EMIC peak remains apparent (panel c). The wave-frequency may be significantly altered in the presence of suprathermal electrons but only at large wave-numbers corresponding to the EFH instability, see the plots in Figs. 13.

\section{DISCUSSIONS AND CONCLUSIONS}

The EMIC fluctuations are regularly observed in the solar wind, but their origin is still unclear. These are small scale fluctuations that can result from a decay of the large scale fluctuation transported by the solar wind, or can be generated locally by the EMIC instability driven by the temperature anisotropy of protons (ions). The EMIC instability is fast enough to constrain the proton anisotropy in a local scenario, but the observations do not conform to the anisotropy thresholds predicted by the early theories with simplified approaches. Recently, Shaaban et al. (2015) have shown that the anisotropic electrons may have important effects on the EMIC instability and this effect is stimulated by the electron/proton temperature ratio. In this paper we have investigated for the first time the effects of the suprathermal electrons on this instability. The fluxes of suprathermal electrons are ubiquitous and highly anisotropic in the solar wind, and may, in general, be more intense than suprathermal protons.

Quantified by the power-index $\kappa_{e}$, the supra-thermal electrons are found to have an important influence on the EMIC instability, significantly enhancing the effects of the anisotropic electrons and the temperature ratio $\Theta$ previously described by Shaaban et al. (2015). Thus, the electrons with $A_{e}>1$ have an inhibiting effect on the EMIC grow-rates (Shaaban et al. 2015), and here we have shown that this effect is enhanced by the suprathermal electrons: growth rates decrease with the decrease of the power-index $\kappa_{e}$. Moreover, the effect of suprathermal electrons is highly dependent on the electron anisotropy, vanishing completely for isotropic electrons $\left(A_{e}=1\right)$. On the other hand, the inhibiting effects of the electron anisotropy and suprathermals increase with the temperature ratio $\Theta$. All these effects are confirmed by the EMIC thresholds derived in Figs. 6 and 7 for very low levels of the maximum growth rates approaching the marginal stability. The EMIC thresholds obtained in this case are increased by the electron anisotropy. This effect is enhanced by the temperature contrast between electrons and protons and by the suprathermal electrons, especially for higher values of the proton plasma beta, i.e., $\beta_{p, \|}>0.1$. Comparison with the observations do not indicate a better alignment of the EMIC thresholds to the limits of the proton anisotropy in the solar wind. However, under the influence of suprathermal electrons, the EMIC thresholds show a tendency to exceed the observational limits of the proton anisotropy, especially for higher values of the plasma beta $\beta_{p, \|}^{M}>1$, where the potential of this instability to constrain the proton anisotropy is significantly increased.

In the opposite case, the anisotropic electrons with $A_{e}<1$ stimulate the EMIC instability, and this effect is enhanced in the presence of suprathermal electrons (i.e., by decreasing the $\kappa$-index). However, a major enhancing effect is more apparent in this case for the $\mathrm{EFH}$ instability that gives rise to a second peak of the growth rates at higher wave-numbers. Thus, the EFH peak may in general exceed the EMIC peak, which becomes indistinguishable for certain conditions. However, the EMIC growth rates are always enhanced by the suprathermal electrons with $A_{e}<1$, leading to the conclusion that the EMIC thresholds are diminished in this case, and are even less relevant for the anisotropy limits of solar wind protons. We must add that all these effects are revealed only by a Kappa approach which assumes the effective temperature of electrons increasing with the increase of suprathermal electrons. Otherwise, for a Kappa model with the effective temperature independent of $\kappa_{e}$, the EMIC instability is not affected by these populations.

Our results in the present paper complement those of a recent series of investigations on the proton anisotropy instabilities, namely, the parallel firehose (Michno et al. 2014), and the EMIC instability (Shaaban et al. 2015). These studies are based on advanced and less-idealized kinetic approaches, which enable us to decode the interplay of thermal (core) protons with thermal and suprathermal electrons, and implicitly their destabilizing effects on different plasma modes. Thus, the parallel firehose instability is found to be markedly affected by the presence of anisotropic electrons, such that the instability thresholds predicted under some circumstances may describe the observations without considering the oblique firehose mode (Michno et al. 2014). The same anisotropic electrons can also change the EMIC thresholds but whithout a 
satisfactory reshaping that could explain the observations (Shaaban et al. 2015). In conclusion, we now can add that suprathermal electrons have an important influence on the EMIC instability, and this influence is highly conditioned by two principal factors: the temperature anisotropy of electrons which may stimulate or inhibit the EMIC fluctuations, and the electron/proton temperature ratio which enhances the effects of the anisotropic electrons. Moreover, the EMIC instability thresholds undergo major changes in the presence of anisotropic electrons with $A_{e}<1$ and an important suprathermal component (i.e., for lower values of $\kappa_{e}$ ), but the new thresholds do not show better alignment to the limits of the proton anisotropy $A_{p}>1$ measured at $1 \mathrm{AU}$ in the solar wind. Apparently negative, these results are however important, as they provide us with enlightening answers, namely, that neither the mutual electron-proton effects analyzed by Shaaban et al. (2015), nor the presence of suprathermal electrons studied in the presence paper can influence the EMIC thresholds to explain the limit of proton anisotropy in the solar wind.

The authors acknowledge the use of WIND SWE (Ogilvie et al. 1995) ion data, and WIND MFI (Lepping et al. 1995) magnetic field data from the SPDF CDAWeb service: http://cdaweb.gsfc.nasa.gov/. The authors acknowledge support from the Katholieke Universiteit Leuven. These results were obtained in the framework of the projects GOA/2015-014 (KU Leuven), G0A2316N (FWO-Vlaanderen), and C 90347 (ESA Prodex 9). The research leading to these results has also received funding from IAP P7/08 CHARM (Belspo), and the European Commission's Seventh Framework Programme FP7-PEOPLE- 2010-IRSES269299 project-SOLSPANET(www.solspanet.eu). This project has received funding from the European Union's Seventh Framework Programme for research, technological development and demonstration under grant agreement SHOCK 284515. S.M. Shaaban would like to thank the Egyptian Ministry of Higher Education for supporting his research activities and and would like to acknowledge the discussions and suggestions of Prof. S. A. Elwakil.

\section{References}

Bale, S. D., Kasper, J. C., Howes, G. G., Quataert, E., Salem, C., Sundkvist, D.: Phys. Rev. Lett., 103, 211101 (2009)

Feldman, W. C., Asbridge, J. R., Bame, S. J., Montgomery, M. D., Gary, S. P.: J. Geophys. Res., 80, 418 (1975)

Fried, B. D., Conte, S. D.: The Plasma Dispersion Function. New York: Academic Press (1961)
Gary, S. P.: Theory of Space Plasma Microinstabilities. Cambridge university press (1993)

Gary, S. P., Lee, M. A.: J. Geophys. Res., 99, 11297 (1994)

Gary, S. P., Montgomery, M. D., Feldman, W. C., Forslund, D. W.: J. Geophys. Res., 81, 1241 (1976)

Gary, S. P., Skoug, R. M., Steinberg, J. T., Smith, C. W.: Geophys. Res. Lett., 28, 2759 (2001)

Hellinger, P., Trávníček, P.: J. Geophys. Res., 110, A04210 (2005)

Hellinger, P., Trávníček, P., Kasper, J. C., \& Lazarus, A. J.: Geophys. Res. Lett., 33, L09101 (2006)

Hellberg, M.A., Mace, R.L., Baluku, T.K., Kourakis, I., Saini, N.S.: Physics of Plasmas, 16, 094701 (2009)

Hellberg, M.A., Mace, R.L., Cattaert, T.: Space Sci. Rev., 121, 127 (2005)

Henning, F. D., Mace, R. L.: Physics of Plasmas, 21, 042905 (2014)

Isenberg, P. A., Maruca, B. A., Kasper, J. C.: Astrophys. J., 773, 164 (2013)

Kennel, C. F., Petschek, H. E.: J. Geophys. Res., 71, 1, (1966)

Kennel, C. F., Scarf, F. L.: J. Geophys. Res., 73, 6149 (1968)

Kourakis, I., Sultana, S., Hellberg, M.A.: Plasma Phys. Control. Fusion 54, 124001, (2012)

Lazar, M., H. Fichtner, and P. H. Yoon.: Astron. Astrophys., in press (arXiv:1602.04132) (2016)

Lazar, M., Poedts, S., Fichtner, H.: Astron. Astrophys., 582, A124 (2015)

Lazar, M.: Astron. Astrophys., 547, A94 (2012)

Lazar, M., Poedts, S., Schlickeiser, R.: Astron. Astrophys., 534, A116 (2011)

Lazar, M., Schlickeiser, R., Shukla, P. K.: Physics of Plasmas, 15, 042103 (2008)

Lepping, R. P., Acŭna, M. H., Burlaga, L. F., et al.: Space Sci. Rev., 71, 207 (1995)

Livadiotis, G., McComas, D.J.: Space Sci. Rev., 175, 183 (2013)

Leubner, M., Schupfer, N.: J. Geophys. Res., 105, 27387 (2000)

Mace, R. L., Sydora, R. D., Silin, I.: J. Geophys. Res., 116, A05206 (2011)

Maksimovic, M., Pierrard, V., Riley, P.: Geophys. Res. Lett., 24, 1151 (1997)

Maksimovic, M., Zouganelis, I., Chaufray, J. Y., Issautier, K., Scime, E. E., Littleton, J. E., et al.: J. Geophys. Res., 110, A09104 (2005)

Matteini, L., Landi, S., Hellinger, P., Pantellini, F., Maksimovic, M., Velli, M., Goldstein, B. E., Marsch, E. Geophys. Res. Lett., 34, L20105 (2007)

Michno, M. J., Lazar, M., Yoon, P. H., Schlickeiser, R.:Astrophys. J., 781, 49 (2014)

Newbury, J. A., Russell, C. T., Phillips, J. L., Gary, S. P.: J. Geophys. Res., 103, 9553 (1998)

Ogilvie, K. W., Chornay, D. J., Fritzenreiter, R. J., et al.: Space Sci. Rev., 71, 55 (1995)

Pierrard, V., Lazar, M.: Sol. Phys., 267, 153 (2010)

Shaaban, M. S., Lazar, M., Poedts, S., \& Elhanbaly, A.: Astrophys. J., 814, 34 (2015)

Štverák, Š., Trávníček, P., Maksimovic, M., Marsch, E., Fazakerley, A. N., \& Scime, E. E.: J. Geophys. Res., 113, A03103 (2008) 
Summers, D., \& Thorne, R. M.: Physics of Fluids B, 3, 1835 (1991)

Vasyliunas, V. M.: J. Geophys. Res., 73, 2839 (1968)

This manuscript was prepared with the AAS LATEX macros v5.2. 